\title{
All-Atom Molecular Dynamics Simulations of
}

\section{Whole Viruses}

\author{
Elvira Tarasova ${ }^{1,2,3}$, Dmitry Nerukh ${ }^{1 *}$ \\ ${ }^{1}$ Department of Mathematics, Aston University, Birmingham, B4 7ET, UK \\ ${ }^{2}$ Laboratory for Computational Molecular Design, RIKEN Center for Biosystems Dynamics \\ (BDR), Building B, 6-2-4 Furuedai, Suita, Osaka 565-0874, Japan \\ ${ }^{3}$ Immanuel Kant Baltic Federal University, A. Nevskogo str. 14, Kaliningrad 236041, Russian \\ Federation
}

*D.Nerukh@aston.ac.uk 


\begin{abstract}
Classical Molecular Dynamics modelling of whole viruses or their capsids in explicit water is discussed and known examples from the literature are analysed. Only works on all-atom modelling in explicit water are included. Physical chemistry of the whole system is the focus that includes the structure and dynamics of the biomolecules as well as water and ions behaviour in and around the virus particle. It was demonstrated that in most investigations molecular phenomena that currently can not be studied experimentally successfully reproduced and explained by the simulations. These include, for example, transport and distribution of ions inside viruses that ultimately connected to their stability, the hydrodynamic pressure in the capsid related to viruses' elastic properties, the role of metal ions in virus swelling, and others. Current and future tendencies in the development of all-atom virus simulations are outlined.
\end{abstract}

\title{
TOC GRAPHICS
}

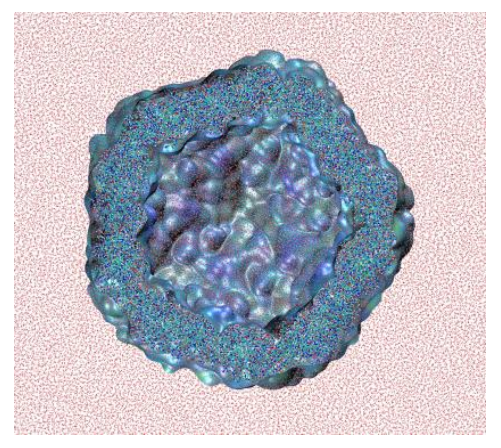

KEYWORDS whole virus structure, whole virus dynamics, phage, bacteriophage, capsid, genome. 
State of the art experimental capabilities to measure the atomistic structure of very large molecular systems (a recent example presents the cryo-EM measured structure of a $125 \mathrm{~nm}$ diameter virus capsid [1]) rely critically on sophisticated modelling techniques that fit known chemical structures to three-dimensional density maps obtained from the experimental data. Currently these modelling techniques are predominantly concerned with structural fitting, which is especially difficult for the parts of the density where ambiguity is possible due to lower resolution or absence of data. However, the atomistic dynamics is very important, both for better quality of fitting (for example, by indicating flexible regions of molecules where experimental data is of poor quality by definition) and for subsequent use of the obtained atomistic models (providing, for example, short lived conformations unobtainable in the experiment). 'Modelling atomistic dynamics' means classical Molecular Dynamics (MD). Therefore, MD simulations of very large, by current MD standards, systems are useful and important despite the technical difficulties caused by the sheer number of atoms in the system.

Among various large molecular systems with known atomistic structure viruses are particularly convenient for MD modelling. This is because they exist in solution as isolated molecular entities, interacting only with water and ions. Complicated and poorly known interactions with other viruses, cell membranes, other cell organelles, etc can be excluded from simulation, at least for some stages of the virus life cycle. Also, the size of viruses varies in large interval, from $\sim 17 \mathrm{~nm}$ in diameter, amenable to rather routine MD simulations, to hundreds of nanometers, currently out of reach for MD. Despite the diversity of their structure and function, it is possible to compile a set of viruses with overall similar construction. For example, they would all consist of nothing else but a capsid formed by a repeating copy of one (or a small group) medium size protein and a genome of relatively short DNA or RNA strand packed inside 
this capsid. A set of such viruses of various sizes can serve as convenient models for tuning and validating MD simulations.

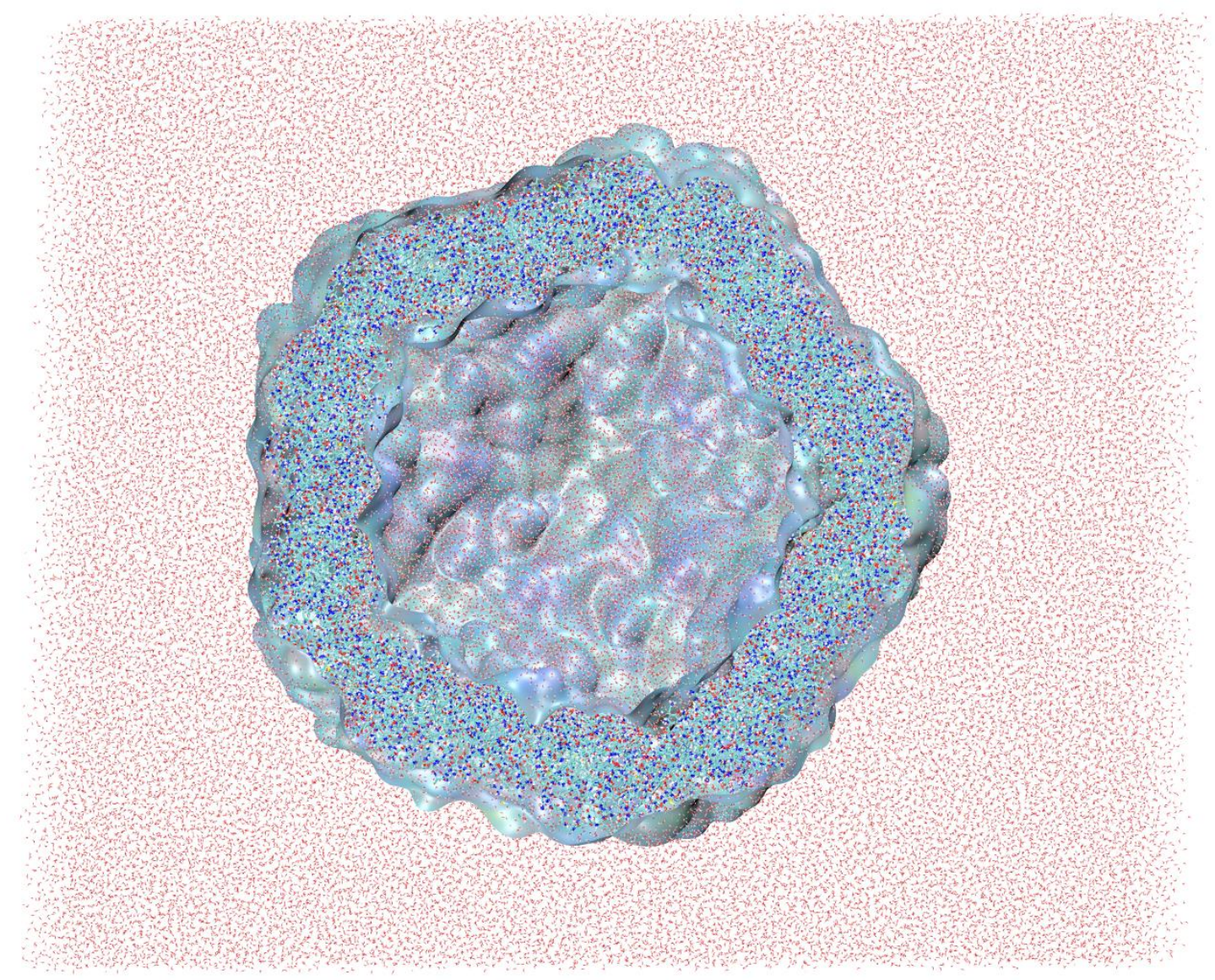

Figure 1 Cross-cut through the all-atom model of PCV2 capsid without genome immersed in a simulation box filled with explicit water [5]

This perspective is devoted to all-atom MD simulations of whole viruses or their complete capsids. Importantly, we consider only works on atomistic modelling, excluding (much more numerous) publications on coarse grained simulations of viruses. We concentrate on the physical chemistry side of modelling rather than the biological aspects of studied viruses. From this point of view the interaction with water and ions is particularly important and it usually 
receives significantly less attention (if any) in biological literature. We, therefore, selected mostly simulations with explicit solvent (Figure 1), that is where water molecules and ions are included in the model at atomistic representation [2], rather than a structureless continuum or any other approximate external correction.

Only few years ago publications on all-atom whole virus modelling with explicit solvent started to appear. The first work was published in 2006 by the Klaus Schulten group [3] where Satellite Tobacco Mosaic Virus (STMV) was modelled. This virus consists of an RNA genome enclosed in an icosahedral capsid of approximately $17 \mathrm{~nm}$ diameter. STMV is a 'non-enveloped' virus that is the virus shell consists of only one layer made of proteins, in contrast to the 'enveloped' viruses where in addition there is another outer layer of lipids and proteins. The capsid was built from 60 copies of a single protein, the initial structure of which was taken from crystallographic measurements. An empty capsid was modelled as well as the whole virus with artificial RNA, built with the assumption that its structure consists of 30 double stranded stems (which backbone was resolvable from the crystallographic data) and arbitrarily packed single stranded regions connecting the resolved double stranded ones [4], the sequence of the RNA was artificial and its length (949 nucleotides) was similar to the native genome (1058). This RNA core was also modelled separately, without the capsid.

The results of modelling showed that water can pass through the capsid's wall. The authors also calculated the diffusion constant of chloride ions in the system. More interesting results for biological applications concerned the stability of the system. The capsid containing RNA and RNA separately turned out to be stable during all time of modelling (13ns and 10ns). However, the empty capsid was unstable, where the protein blocks shifted towards the capsid's centre that lead to overall deformation of the capsid. The fact of unstable empty capsid agreed with 
experiment. These findings had implications for virus assembly mechanism emphasising the role of RNA in the process.

For the stable entire virion, an interesting observation on the dynamics of the protein units was found. The units around an equatorial 'belt' exhibited correlated motion, while the units at the opposite ends were in anticorrelated motion. This is one of the results that was possible to obtain only in all-atom MD of an entire virus.

The results of the publication allowed to conclude that the correct assembly of the virus proceeds in the presence of RNA, while empty capsids are not formed. We have also observed this behaviour of capsid deformation for a different virus PCV2 [5] when the genome is absent inside the capsid. We have shown that such instability is caused by incorrect distribution of ions on the capsid's surfaces (inside and outside). In contrast to STMV, however, empty capsids of PCV2 are known to exist experimentally [6].

Three years later after the first publication a system of 4.5 million atoms was modelled [7], with a focus on mechanical properties of the capsid of Southern Bean Mosaic Virus. In this work, the Force Probe MD simulation was applied to obtain the distribution of mechanical properties (elastic constant and yielding forces) on viral capsid at atomistic level. The approach allowed to analyse the viral shell properties with the variation of the probe velocity of the forcing tip-sphere and its size. The authors observed three deformation regimes under the load. They also did not find any water flow through the capsid wall even under tip-sphere indentation that resulted in substantial change of the inside volume.

It is generally assumed that the removal of calcium ions from the capsid's structure of Southern Bean Mosaic Virus leads to its swelling, which is the initial stage of cell infection 
during which the genome is released [8]. In their subsequent publication the authors [9] continued the investigation of the capsid's mechanical properties of the virus with the Force Probe MD simulation at atomistic resolution with the aim to investigate how the mechanical properties of the capsid are changed when calcium is removed. They also investigated the distribution of the elastic constant and yielding forces on the outer surface as well as on the inner surface of the capsid, which is not possible to do experimentally for the inner surface. In the work they noted that after calcium removal the mechanical properties changed before the changes in the structure. The found weakening along the 5-fold axes of the capsid inspired the hypothesis that the pentamers that form the capsid's structure serve as possible ports for RNA release.

For some viruses the increase of their diameter or 'swelling' was observed when metal ions (for example, $\mathrm{Ca}^{2+}$ ) were removed from the capsid's structure [10-11]. This property plays an important role in the infection process. MD modelling allows studying the dynamics and the structural features of the capsid connected with swelling, as it was done in [12] for another satellite virus, Satellite Tobaco Necrosis Virus (STNV). Modelling was performed for capsids containing structural $\mathrm{Ca}^{2+}$ ions and the capsids after the removal of ions in explicit water solutions for 1 microsecond each, RNA was not included into the simulation.

The authors analysed how the water permeability of the capsid changes after calcium removal and they found that the capsid's calcium-binding sites play different roles in the STNV structure: some are responsible for the swelling after removing the calcium ions, while others play structural role and do not take part in swelling.

In [13] the authors performed the MD simulation of the native and swollen states of the Cowpea Chlorotic Mottle Virus (CCMV) in vacuum and showed that the study of structural 
transitions in the swollen capsid requires long time simulation. They applied their multiscale molecular dynamics/order parameter extrapolation (MD/OPX) approach [14] for the long time (200 ns) simulation of the swollen CCMV capsid in vacuum that allowed to investigate its structural transition mechanisms.

In their next work [15], the authors demonstrated the application of their methodology MD/OPX to the investigation of the CCMV capsid swelling in solution mimicking environment. Their simulation results showed that the swelling of the capsid, as well as the shrinking notable in simulation in vacuum, was observed accompanied by the loss of symmetry first locally and then in the whole capsid.

The conformation of unstructured, dynamic fragments of the capsid are impossible to measure experimentally, but they can be reconstructed, and their structure and dynamics can be studied in simulation as this was done in [16] for poliovirus. The poliovirus capsid includes four proteins VP1, VP2, VP3, VP4, that form a single structural unit (block), 60 copies of this unit make the whole capsid. X-ray analysis could not determine the structure of the first 20 aminoacids of the N-terminal domain of VP1, which is involved in the infection process. Modelling was performed for four systems: whole virus with RNA, empty capsid, pentamer, and isolated N-terminal domain. The size of the modelled systems reached 4 million atoms. The modelling was performed for $10 \mathrm{~ns}$ for each system in $\mathrm{NaCl}$ solution of $0.154 \mathrm{mM}$ concentration. Also, in the system with RNA, $\mathrm{Mg}^{2+}$ were added for stabilising the structure. It was observed that in the detached domain the $\alpha$-helix structure is preserved for the whole $10 \mathrm{~ns}$ of simulation, this domain is more flexible in the empty capsid than in the complete virus. The behaviour of ions in full virion was investigated and it was found that $\mathrm{Na}^{+}$and $\mathrm{Cl}^{-}$ions diffuse across the capsid wall 
through a small crack in the two-fold axis region. $\mathrm{Na}^{+}$diffuse inwards, $\mathrm{Cl}^{-}$diffuse outwards, while $\mathrm{Mg}^{2+}$ remain bound by the RNA.

The properties of this virus were also investigated in 2014 at longer times, up to 200ns [17]. In their work the authors used sodium phosphate buffer at $\mathrm{pH} 7.4$ for reproducing experimental conditions. The total system size was 6.5 million atoms. After long equilibration for $100 \mathrm{ns,}$ water molecules diffusion through the capsid's wall was observed. At equilibrium the time for total replacing of water inside the capsid with the outside water was $25 \mu \mathrm{s}$. This result explains experimental data on the ability of poliovirus to withstand high pressure and pressure shocks [18], water diffuses fast enough to equalise the pressure without destroying the capsid. Results were also obtained on the possibility of water evaporation from the inside of the capsid. These results are important for developing methods of viral deactivation. Despite water diffusion, ions did not pass through the capsid for all $200 \mathrm{~ns}$ of simulation time. This classifies the capsid as a semipermeable membrane. Negative pressure was observed inside the empty capsid that was explained by coulombic interaction of the solution inside the capsid with the capsid excess charges.

Similar results on the capsid functioning as a semipermeable membrane were found in [19] for porcine circovirus type 2 (PCV2). In the work an all-atom modelling of an empty PCV2 capsid in environment similar to physiological with explicit water model was conducted. The capsid was modelled by two methods: the classical MD and a hybrid MD/hydrodynamics approaches. The system size was 1.9 million atoms and the simulation time was $10 \mathrm{~ns}$. It was found that the capsid was stable after $10 \mathrm{~ns}$. The water and ion flows were analysed that showed that while water could cross the capsid wall in both directions, the ions could not. 
In [20] the authors modelled an empty capsid of the HIV-1 virus and investigated its physical chemical properties. The simulated time was $1.2 \mu$ s and the system size reached 64 million atoms. The modelling was performed at physiological conditions with $150 \mathrm{mM} \mathrm{NaCl}$ explicit water solution. The authors investigated the stability of the capsid, both overall and locally, calculated the rate of water exchange between the inside and outside of the capsid, determined the location of the ions on the capsid's surfaces. Electrostatic properties as well as acoustic properties and modes were analysed.

It was found that the capsid can translocate ions with high specificity, that could play a role during the infection by assisting in filtering particular molecules. The sodium ions were transferred through the capsid wall via special channels. It was found that the chloride ions form a layer on the inner surface of the capsid, while sodium ions - on the outer surface. Similar layers were found on the surfaces of PCV2 [5].

A large part of the analysis was devoted to the acoustic modes on the surface of the capsid. Oscillatory behaviour was found that propagates through the capsid similar to capillary waves common to membranes and fluids. Interestingly, the frequencies of these waves were found to lie in the ultrasound region (2.38 to $11.9 \mathrm{MHz}$ ). Moreover, waves of different frequencies were found to be localised at different regions of the capsid (for example, the highest frequency was associated with the tip of the capsid, etc.). It was hypothised that this behaviour plays important role in the infection process as it facilitates the transfer of information through very large distances.

All-atom MD simulation was performed for Hepatitis B virus capsid [21]. The simulations were performed for $1.1 \mu \mathrm{s}$ in explicit water and $150 \mathrm{mM} \mathrm{NaCl}$, thus the size of the system was 6 
million atoms. The results demonstrate high flexibility of the capsid and the acceptance of asymmetric distortion that may result in the limitation of structural determination with Cryo-EM. The HBV capsid is also permeable for water molecules, sodium ions can move through the capsid wall faster than chloride ions. The authors assumed that this slow translocation of the chloride ions may play important function in gradualness in structural distortion of the capsid. Ions of physiological solution could play important part in mediating specific interactions. At the moment all-atom modelling of viruses using MD is at the beginning of active development, which is testified by a small number of publications in the field. These works demonstrate successful application of MD to whole viruses, the analysis of their stability at various conditions, interaction with the environment and, in some rare cases, with the genome. The developed models allow investigations of macroscopic properties of viruses at some stages of their life cycle starting from the fundamental microscopic dynamics. The fact of the existence of stable empty capsids is important as it suggests that such capsids could exist in nature under proper conditions and, perhaps, play role in the virus self-assembly. However, since modelling of such large systems is a new field of research, it is important to pay close attention to correct preparation of the system.

One of the most intriguing questions in this field for both experimentalist and modellers is the structure of the genome inside the capsid and its interaction with the capsid. To date there are very few attempts to reconstruct the genome because experimental data on its structure is fragmented at best. However, rapid progress in structure determination gives us the reason to believe that all-atom modelling of the genome in viruses will be one of the most actively developed directions of research for the next few years. 
The questions of viral self-assembly [22-23] remain largely open. They are related to the analysis of specific interactions between the capsid's units as well as between the genome and the capsid. Such interactions are of key importance for the process of self-assembly and genome packing. All-atom MD modelling will be the instrumental methodology in studying the details of these interaction thus, indirectly, contributing significantly to reconstructing the biological mechanisms of viral self-assembly. Research activity in this direction is likely to be the trend in all-atom MD of viruses.

Overall, all-atom modelling of entire viruses in explicit water has proven its usefulness but at the moment it is at the beginning of its active use. The tendencies in experimental techniques, computer developments, and questions posed by state of the art biology are such that these simulations are likely to see active developments in the near future.

\section{ACKNOWLEDGMENT}

E.T. is a JSPS International Research Fellow, grant number PE17023; D.N. acknowledges the support of JSPS through BRIDGE Fellowship, grant number BR170303.

\section{REFERENCES}

1. Yuan, S.; Wang, J.; Zhu, D.; Wang, N.; Gao, Q.; Chen, W.; Tang, H.; Wang, J.; Zhang, X.; Liu, H.; Rao, Z.; Wang, X. Cryo-EM structure of a herpesvirus capsid at 3.1 Å. Science. 2018, $360,1-11$.

2. Nerukh, D.; Karabasov, S. Water - Peptide Dynamics during Conformational Transitions. J. Phys. Chem. Lett., 2013, 4 (5), 815-819. 
3. Freddolino, P.L.; Arkhipov, A.S.; Larson, S.B.; McPherson, A.; Schulten, K. Molecular dynamics simulations of the complete satellite tobacco mosaic virus. Structure. 2006, 3, 437449.

4. Larson, S.B.; Mcpherson, A. Satellite tobacco mosaic virus RNA: structure and implications for assembly. Curr Opin Struct Biol. 2001, 11, 59-65.

5. Tarasova, E.; Farafonov, V.; Khayat, R.; Okimoto, N.; Komatsu, T. S.; Taiji, M.; Nerukh, D. All-Atom Molecular Dynamics Simulations of Entire Virus Capsid Reveal the Role of Ion Distribution in Capsid's Stability. J. Phys. Chem. Lett. 2017, 8, 779-784.

6. Fan, H.; Ju, C.; Tong, T.; Huang, H.; Lv, J.; Chen, H. Immunogenicity of empty capsids of porcine circovius type 2 produced in insect cells. Vet. Res. Commun. 2007, 31, 487-496.

7. Zink, M.; Grubmüller, H. Mechanical properties of the icosahedral shell of southern bean mosaic virus: A molecular dynamics study. Biophys. J. 2009, 96, 1350-1363.

8. Hsu, C.H.; Sehgal, O.P.; Pickett, E.E. Stabilizing effect of divalent metal ions on virions of southern bean mosaic virus. Virology. 1976, 69, 587-595.

9. Zink, M.; Grubmüller, H. Primary changes of the mechanical properties of Southern Bean Mosaic Virus upon calcium removal. Biophys J. 2010, 17; 98, 687-695

10. Speir, J.A.; Munshi, S.; Wang, G.; Baker, T.S.; Johnson, J.E. Structures of the native and swollen forms of cowpea chlorotic mottle virus determined by X-ray crystallography and cryoelectron microscopy. Structure. 1995, 3, 63-78.

11. Jones, T.A.; Liljas, L. Structure of satellite tobacco necrosis virus after crystallographic refinement at 2.5 Å resolution. J. Mol. Biol. 1984, 177, 735-767.

12. Larsson, D. S. D.; Liljas, L.; van der Spoel, D. Virus capsid dissolution studied by microsecond molecular dynamics simulations. PLoS Comput. Biol. 2012, 8, 1-8. 
13. Miao, Y.; Ortoleva, P.J. Viral structural transition mechanisms revealed by multiscale molecular dynamics/order parameter extrapolation simulation. Biopolymers. 2010, 93, 61-73.

14. Miao, Y.; Ortoleva, P.J. Molecular Dynamics/Order Parameter EXtrapolation (MD/OPX) for Bionanosystem Simulations. J. Comput. Chem. 2009, 30, 423-437.

15. Miao, Y.; Johnson, J.E.; Ortoleva, P.J. All-Atom Multiscale Simulation of Cowpea Chlorotic Mottle Virus Capsid Swelling. J. Phys. Chem. B, 2010, 114, 11181-11195.

16. Roberts, J.A.; Kuiper, M.J.; Thorley, B.R.; Smooker, P.M.; Hung, A. Investigation of a predicted N-terminal amphipathic $\alpha$-helix using atomistic molecular dynamics simulation of a complete prototype poliovirus virion. J. Mol. Graph. Model. 2012, 38, 165-173.

17. Andoh, Y.; Yoshii, N.; Yamada, A.; Fujimoto, K.; Kojima, H.; Mizutani, K.; Nakagawa, A.; Nomoto, A.; Okazaki, S. All-atom molecular dynamics calculation study of entire poliovirus empty capsids in solution. J. Chem. Phys. 2014, 141, 165101.

18. Kingsley, D.H.; Hoover, D.G.; Papafragkou, E.F.I.; Richards, G. P. Inactivation of Hepatitis A Virus and a Calicivirus by High Hydrostatic Pressure. J Food Prot. 2002, 65, $1605-$ 1609.

19. Tarasova, E; Korotkin, I.; Farafonov, V.; Karabasov, S.; Nerukh, D. Complete virus capsid at all-atom resolution: simulations using Molecular Dynamics and hybrid Molecular Dynamics/hydrodynamics methods reveal semipermeable membrane function. J.Mol.Liq. 2017, $245,109-114$.

20. Perilla, J. R.; Schulten, K. Physical properties of the HIV-1 capsid from all-atom molecular dynamics simulations. Nat. Commun. 2017, 8, 15959. 
21. Hadden, J.A.; Perilla, J.R.; Schlicksup, C.J.; Venkatakrishnan, B.; Zlotnick, A.; Schulten, K. All-atom molecular dynamics of the HBV capsid reveals insights into biological function and cryo-EM resolution limits. Elife. 2018, 7, e32478.

22. Jiang, J.; Yang, J.; Sereda, Y.V.; Ortoleva, P.J. Early stage P22 viral capsid self- assembly mediated by scaffolding protein: atom resolved model and MD simulation. J. Phys. Chem. B, $2015,119,5156-5162$.

23. Moerman, P.; Paul van der Schoot; Kegel, W. Kinetics versus Thermodynamics in Virus Capsid Polymorphism. J. Phys. Chem. B. 2016, 120, 6003-6009. 


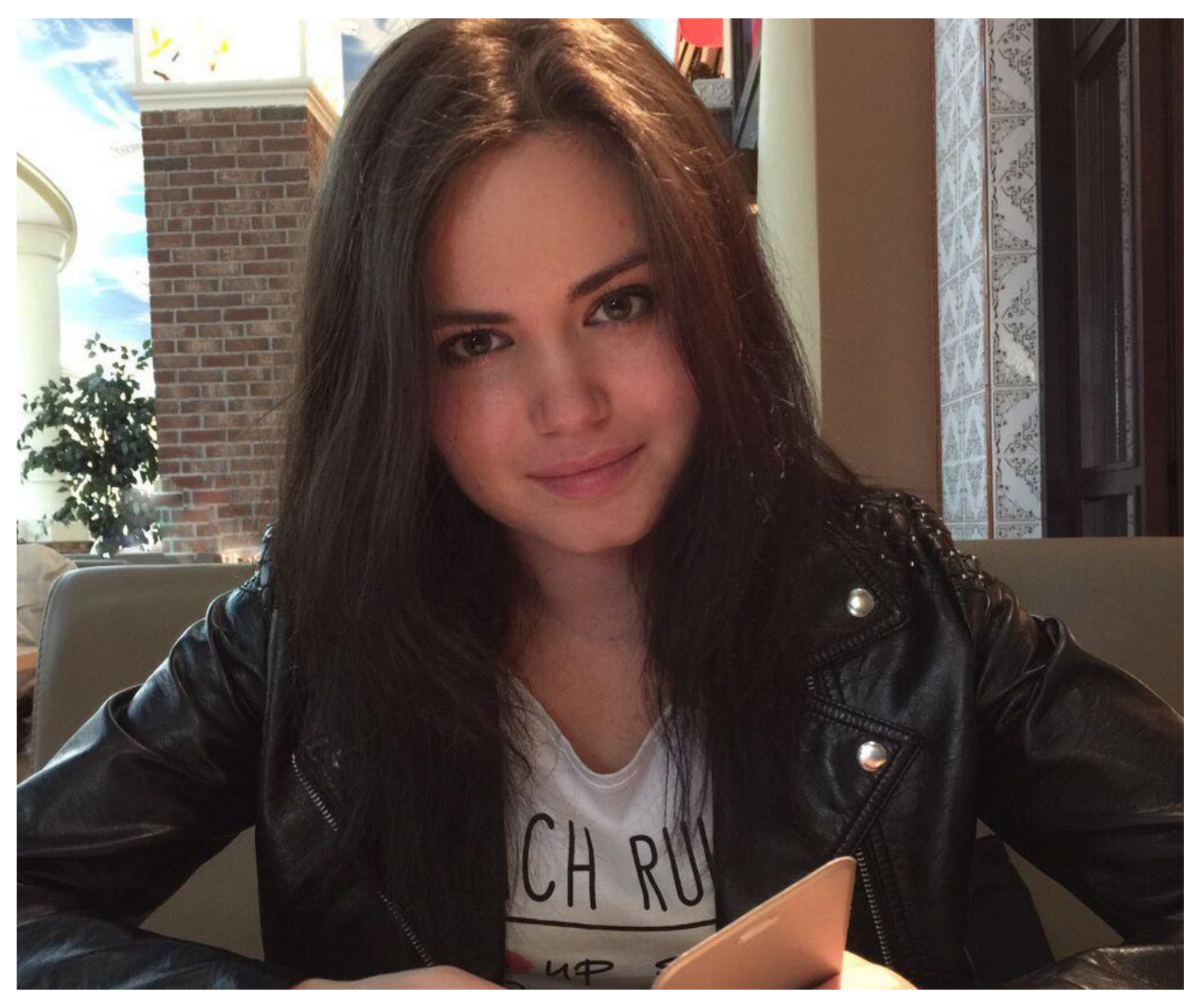

Elvira Tarasova ( $\mathrm{PhD}$ in physical chemistry, 2018) works as a JSPS postdoctoral fellow at RIKEN Institute in Japan. She also did research at Immanuel Kant Baltic Federal University, Russia and Aston University, UK. Her scientific interests are in computational chemistry of biomolecules, including very large systems such as viruses, drug discovery for antimicrobial resistance, and physicochemical properties and mechanisms of viral life cycle. 


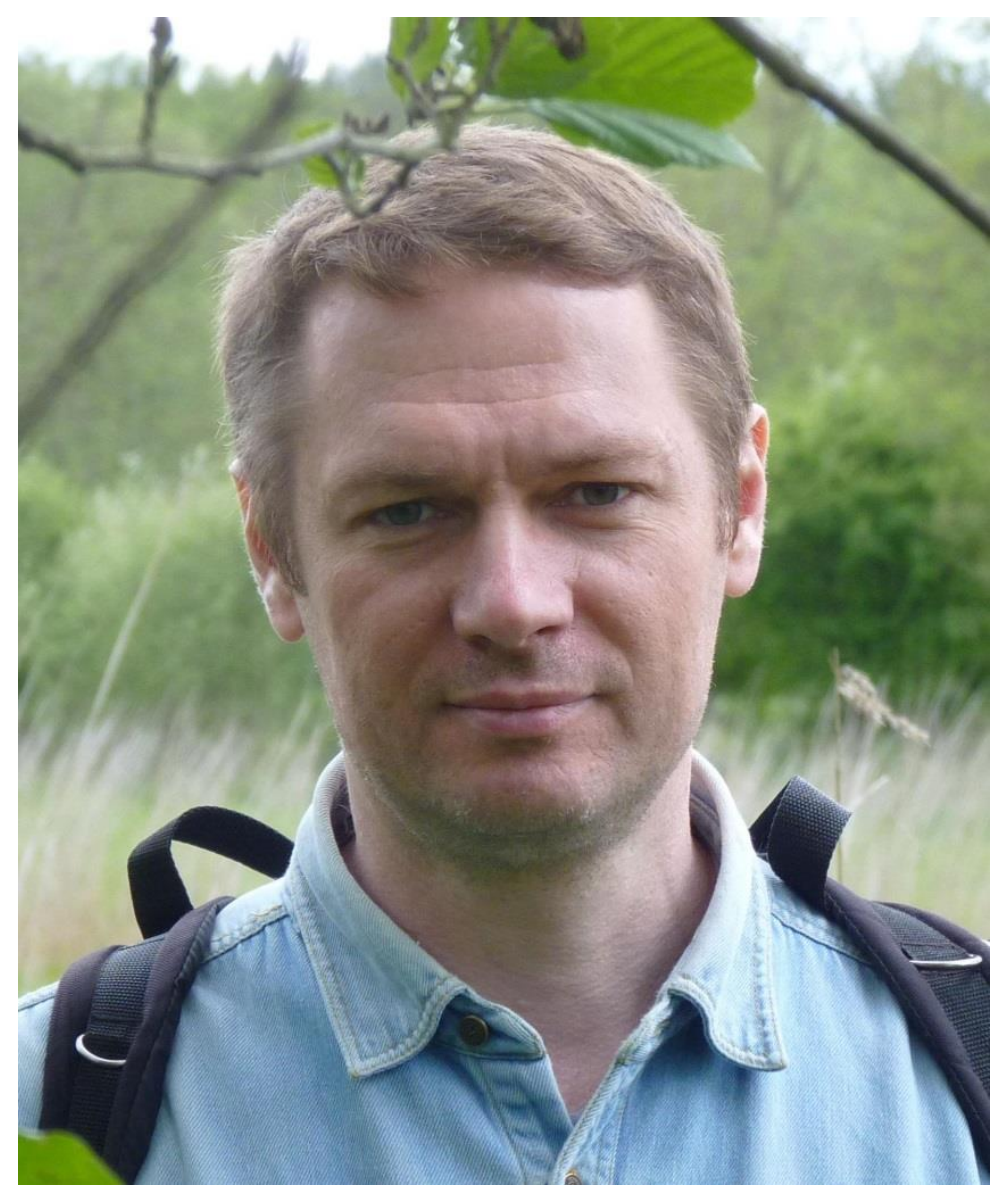

Dmitry Nerukh. After a number of postdoctoral positions at various Universities in the UK and USA, Dr Nerukh worked at Department of Chemistry, Cambridge University, UK, before accepting permanent position at Aston University. His work has led to the development of an original numerical method for Bohmian quantum dynamics and fundamentally new approaches for the complex dynamics of classical molecular systems that applies state of the art mathematical theory of complexity of dynamical systems. His most recent research includes the development of multiscale hybrid Molecular Dynamics / Hydrodynamics framework for modelling large biomolecular systems at several spatial and temporal scales simultaneously. Such systems include all-atom models of entire viruses in solution which are modelled with very large number of atoms using high performance supercomputers. 


\section{PULL QUOTES}

"... works demonstrate successful application of MD to whole viruses, the analysis of their stability at various conditions, interaction with the environment and, in some rare cases, with the genome. "

"One of the most intriguing questions in this field for both experimentalist and modellers is the structure of the genome inside the capsid and its interaction with the capsid" 